\title{
Sistema híbrido basado en redes neuronales artificiales y descomposición modal empírica para la evaluación de la interrelación entre la irradiancia solar total y el calentamiento global
}

\author{
Eric Alberto Suárez-Gallareta ${ }^{1}$, Jorge Javier Hernández-Gómez ${ }^{2}$, \\ Gerardo Cetzal-Balam ${ }^{1}$, Mauricio Gabriel Orozco-del-Castillo ${ }^{1}$, \\ Mario Renan Moreno-Sabido ${ }^{1}$, Raúl Alberto Silva-Aguilera ${ }^{3}$ \\ 1 Instituto Tecnológico de Mérida, \\ Departamento de Sistemas y Computación, \\ México \\ 2 Instituto Politécnico Nacional, \\ Centro de Desarrollo Aeroespacial, \\ México \\ 3 Universidad Nacional Autónoma de México, \\ Instituto de Ciencias del Mar y Limnología, \\ México \\ \{ericsuarezgallareta1996,gerardoce23,xacdc12\}@gmail.com, \\ mauricio.orozco@itmerida.edu.mx, \\ jjhernandezgo@ipn.mx, raul.s@ciencias.unam.mx
}

\begin{abstract}
Resumen. El calentamiento global o cambio climático es probablemente el mayor reto científico actual de la humanidad. De todos los factores tanto naturales como antropogénicos involucrados en el calentamiento global, así como sus complejas interrelaciones, poca atención se ha centrado en los factores externos al sistema Tierra, como lo es la variabilidad solar. En este trabajo se presenta un sistema híbrido de inteligencia artificial basado en redes neuronales artificiales y descomposición modal empírica para determinar la interrelación entre la irradiancia solar total recibida en la Tierra en las últimas cuatro décadas con un índice clave en el cambio climático: la temperatura superficial del mar. Los resultados hasta el momento muestran una evidente interrelación entre ambos índices, sugiriendo que el principal motor de la variabilidad en la temperatura superficial del mar son las variaciones en la entrada de energía solar al sistema Tierra.
\end{abstract}

Palabras clave: Redes neuronales artificiales, descomposición modal empírica, cambio climático, calentamiento global, irradiancia solar, inteligencia artificial, reconocimiento de patrones, temperatura superficial del mar, IA, RNA, DME. 


\title{
Hybrid System based on Artificial Neural Networks and Empiric Modal Decomposition for the Assessment of the Interrelation between Total Solar Irrandiance and Global Warming
}

\begin{abstract}
Global warming or climate change is probably the current greatest scientific challenge that mankind faces. From all both natural and anthropogenic factors involved in global warming, as well as their complex interrelations, few attention has focused on those factors external to Earth, as the solar variability. In this work we present a hybrid system in artificial intelligence based on artificial neural networks and empirical mode decomposition to determine the interrelation between total solar irradiance received by Earth in the last four decades with a key index in climate change: oceanic surface temperature. The results of this ongoing research show an evident interrelation between both indexes, and strongly suggest that the main external factor driving of the variations in the oceanic surface temperature are the variations in the input solar energy of Earth.
\end{abstract}

Keywords: Artificial neural networks, empirical mode decomposition, climate change, global warming, solar irradiance, artificial intelligence, pattern recognition, oceanic surface temperature, AI, ANN, EMD.

\section{Introducción}

Actualmente, es probable que no haya mayor reto científico en el mundo que el que representa el calentamiento global. Desde la mitad del siglo XIX se empezaron a notar anomalías en los registros de temperatura alrededor del mundo, que empezaron a marcar una tendencia clara de aumento a partir de la década de los 1950s. Estos registros junto con registros paleoclimáticos de los últimos miles de años constituyen una prueba fehaciente del calentamiento global 25]. A raíz de la preocupación de las tendencias del calentamiento global en los 1980s, países miembros de la Organización Meteorológica Mundial (OMM) y el Programa de las Naciones Unidas para el Medio Ambiente (PNUMA) establecieron en 1988 el Panel Intergubernamental del Cambio Climático, conocido por el acrónimo en inglés IPCC (Intergovernmental Panel on Climate Change) 24, mismo que fue posteriormente ratificado por la Asamblea General de la Organización de las Naciones Unidas (ONU) mediante la Resolución 43/53. El IPCC publica informes especiales de evaluación del cambio climático basados en información científica, técnica y socioeconómica actual sobre el riesgo que representa, sus potenciales consecuencias medioambientales y socioeconómicas, así como las posibles opciones de adaptabilidad o mitigación de sus efectos.

El calentamiento global se define como el aumento de la temperatura global media del aire sobre la Tierra y el océano 25, y en el último informe del IPCC se destaca que si bien en 2013 el aumento en la temperatura global media fue del orden de 0.4 C (respecto a la media de temperatura de 1961 a 1980), se advierte 
que para el año 2100 la tendencia podría superar los $2.5 \mathrm{C}$ con consecuencias catastróficas tanto para la humanidad como para el planeta y la vida en él (Fig. 1).

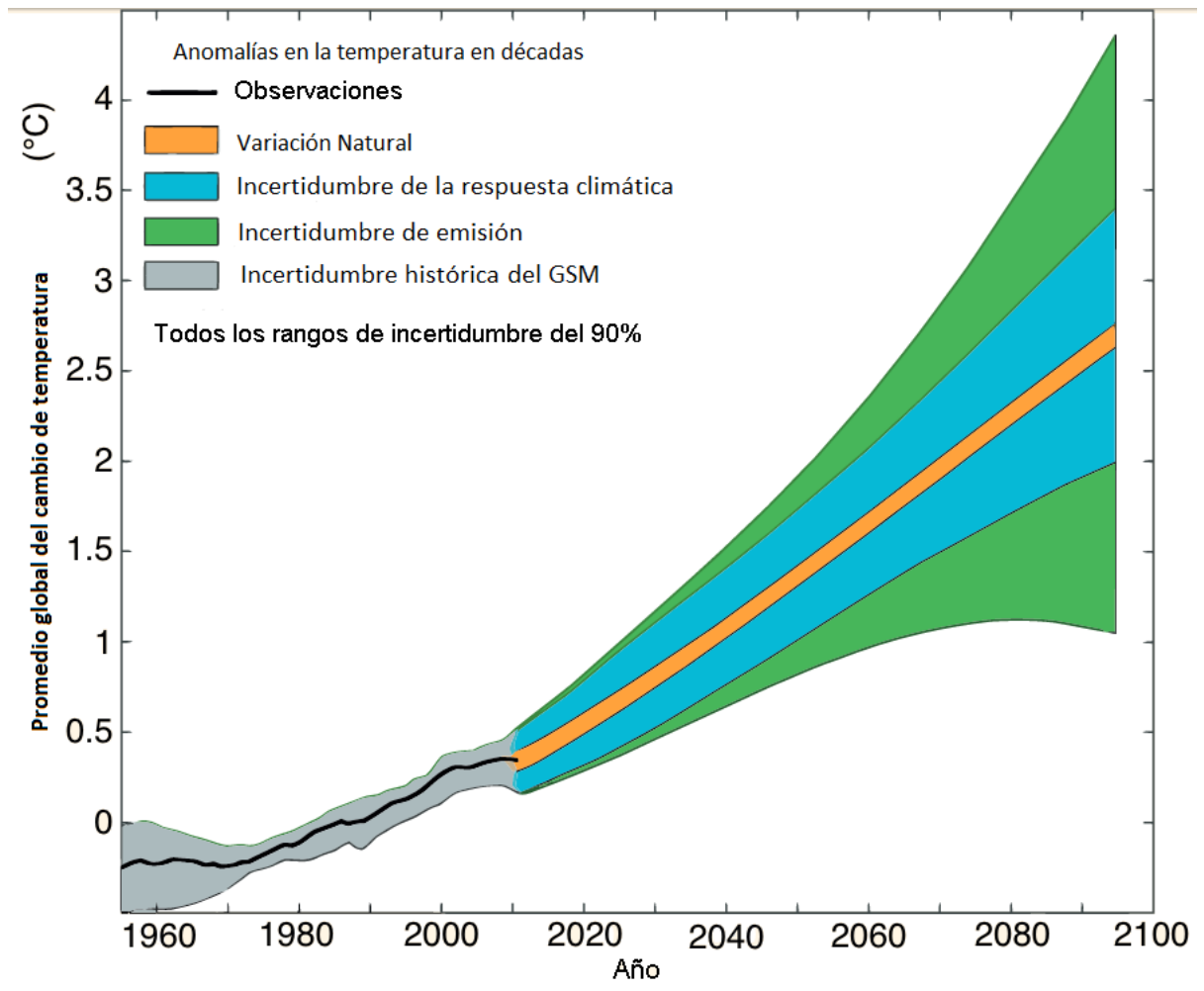

Fig. 1. Diagrama esquemático del cambio en la temperatura media superficial (C) por décadas de registros históricos (línea negra) con sus incertidumbres históricas (gris), junto con proyecciones futuras del clima y sus incertidumbres 25]. Los valores están normalizados con valores medios de 1961 a 1980. La variabilidad natural (naranja) se deriva del modelo de variabilidad interanual y se supone constante en el tiempo. La incertidumbre en las emisiones (verde) se estima como la diferencia media del modelo en proyecciones para diversos escenarios. La incertidumbre en la respuesta del clima (azul, sólida) se basa en la dispersión del modelo climático, con incertidumbres agregadas del ciclo del carbón, así como con estimados gruesos de incertidumbres adicionales de algunos procesos pobremente modelados 19 .23.

Aunque son incontables los procesos fisicoquímicos involucrados en el calentamiento global, así como complejas y altamente no lineales sus interrelaciones, es indudable que el aumento de la temperatura global media se debe a que el balance global de energía del sistema Tierra ha sufrido modificaciones en las 
últimas décadas, de modo que el contenido de calor del planeta ha aumentado. Esto podría deberse a una mayor entrada de energía al planeta, o bien, a una disminución en su disipación energética. Sin embargo, la mayoría de la investigación respecto al tema del cambio climático se centra, o bien en el estudio de dichos procesos fisicoquímicos per se, en sus causas y consecuencias antropogénicas, o bien en la realización de proyecciones o predicciones del comportamiento de la temperatura global media en diversos escenarios. No obstante, y a pesar de la importancia de tener predicciones confiables, la gran mayoría de la investigación al respecto se basa en construir modelos, ya sean fenomenológicos o de primeros principios, acerca del clima regional o global.

Estos modelos se suelen dividir en Modelos de Circulación General AtmosféricoOceánicos, Modelos del Sistema Tierra 12, Modelos del Sistema Tierra de

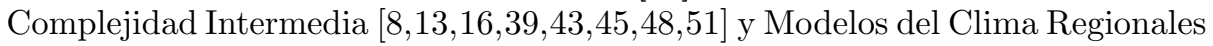
[31.44. Para una revisión exhaustiva de los modelos climáticos, ver 25].

Claramente, el calentamiento global es un tema delicado que podría beneficiarse ampliamente del poder predictivo de las técnicas de la inteligencia artificial para el reconocimiento de patrones. Todos los procesos internos en el sistema Tierra, tanto naturales como antropogénicos que contribuyen al cambio climático, afectan principalmente a la salida de energía del planeta y además poseen complejas interrelaciones no lineales. De esta manera, vale la pena observar los procesos que afectan la entrada energética al planeta. Dentro de ellos puede estar la variabilidad solar, que puede tomar escalas centenarias o inclusive milenarias 21], variaciones en los alineamientos astronómicos entre el sol y la tierra (como los ciclos de Milankovitch), así como por ejemplo impactos de asteroides mayores 25]. De todos estos fenómenos, el mayor forzamiento térmico natural sobre la Tierra es indiscutiblemente la variabilidad solar, misma que ha probado ser multiperiódica 26]. Existen diferentes índices que pueden dar cuenta de la variabilidad solar como lo son mismos los ciclos solares [4, 38, 40], las manchas solares [17, 41], la presencia de Ground Level Enhancements en los rayos cósmicos solares [2:| 6 |38 46], el viento solar y el índice geomagnético [14 34], el campo magnético solar [37], entre otros.

Sin embargo, de todos estos, probablemente el de mayor relevancia para el problema del calentamiento global sea la irradiancia solar total (IST) 25], que es la potencia solar recibida por unidad de área (energía solar recibida por unidad de tiempo y área), pues la IST es la mayor contribución de entrada de energía térmica al sistema Tierra. De esta forma, el tener predicciones confiables del comportamiento futuro de la IST es fundamental para proyectar los balances de energía térmica al futuro y así tener evaluaciones más precisas de las posibles tendencias de la temperatura global media en diversos escenarios a corto, mediano y largo plazo. Cabe destacar que todas las mediciones de la ITS coinciden en la existencia de periodicidad en este índice [29].

En este trabajo, actualmente en proceso, se propone la implementación de un método híbrido en inteligencia artificial constituido por una Red Neuronal Artificial (RNA) junto con el método de Descomposición Modal Empírica (DME) para el reconocimiento de patrones dentro de datos de IST obtenidos de Agencia 
Nacional Oceánico-Atmosférica (NOAA por sus siglas en inglés) de los Estados Unidos de Norteamérica. Para este estudio, se utilizó la base de datos de valores compuestos de la IST en un periodo de 1978 al 30 de junio de 2017 [ (Fig. 2. Este sistema híbrido se encarga de buscar relaciones entre la IST y otros indicadores fuertemente relacionados con el cambio climático, en este caso, la Temperatura Superficial del Mar (TSM).

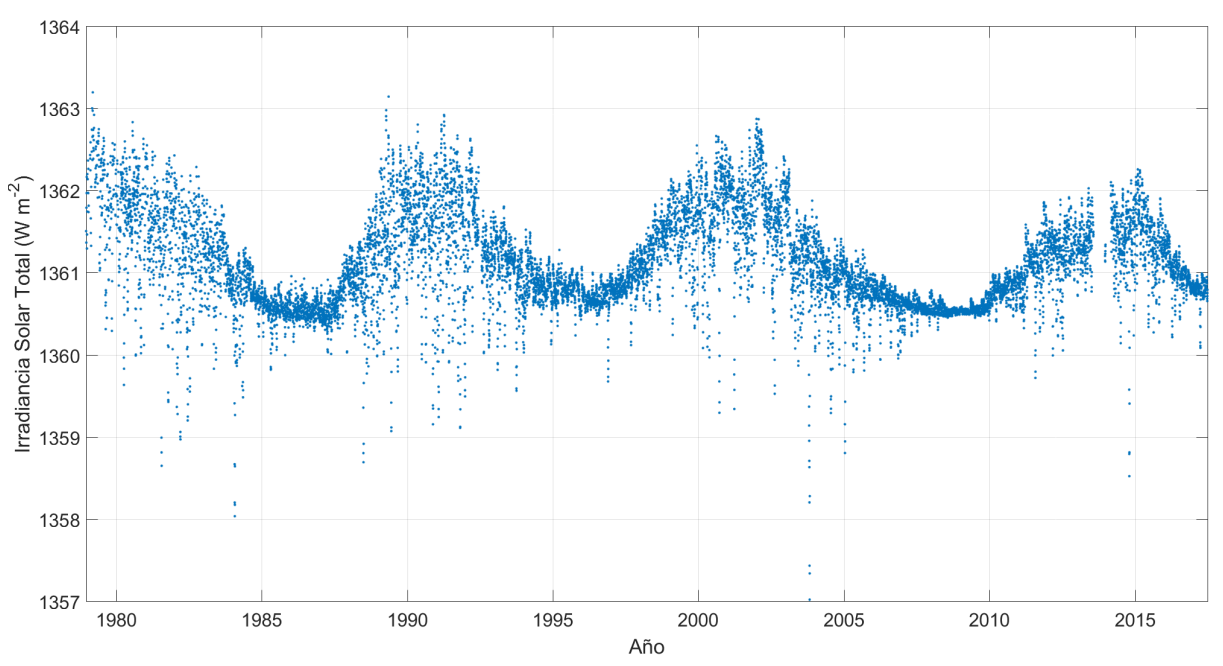

Fig. 2. Datos de irradiancia solar total obtenidos de la Agencia Nacional OceánicoAtmosférica de los Estados Unidos de Norteamérica en un periodo de 1978 al 30 de junio de 2017 [.

\section{Metodología}

A continuación, se presentan los métodos utilizados en este estudio. Debido a que la base de datos diaria de la IST [9] tiene valores faltantes, estos son interpolados con el método de kriging 30,33 .

\subsection{Método de kriging}

El método de kriging (krigeaje o krigeado) es un procedimiento geoestadístico de interpolación avanzado que genera una superficie estimada a partir de un conjunto de puntos dispersados. El algoritmo presupone que la distancia o la dirección entre los puntos de muestra refleja una correlación espacial que puede utilizarse para explicar la variación en la superficie. La herramienta ajusta una función matemática a una cantidad especificada de puntos o a todos los puntos 
dentro de un radio específico para determinar el valor de salida para cada ubicación. El método consiste primeramente en el análisis estadístico exploratorio de los datos, el modelado de variogramas, la creación de la superficie y (opcionalmente) la exploración de la superficie de varianza. Este método es más adecuado cuando se sabe que hay una influencia direccional o de la distancia correlacionada espacialmente en los datos, como es el caso de los datos en la Fig. 2. El método kriging pondera los valores medidos circundantes para calcular una predicción de una ubicación sin mediciones mediante la ecuación (1):

$$
\hat{Z}\left(s_{0}\right)=\sum_{i=1}^{N} \lambda_{i} Z\left(s_{i}\right) .
$$

El método de kriging se ha usado extensivamente tanto en las ciencias naturales como directamente en las matemáticas aplicadas y ciencias de la computación [32 50]. Una vez interpolados los datos, se procedió a determinar las tendencias y componentes modales principales en la serie de datos (Fig. 2) mediante la DME.

\subsection{Descomposición modal empírica}

El algoritmo de DME, presentado por primera vez en 1998 [22], se basa en producir envolventes lisos definidos por máximos y mínimos locales de una secuencia y substracción subsiguiente de la media de estas envolventes a partir de la secuencia inicial. Esto requiere la identificación de todos los extremos locales que están conectados además por líneas spline cúbicas para producir los envolventes superior e inferior 28] Es un método adaptivo de análisis adecuado para el procesamiento de series que son no estacionarias y no lineales. DME realiza operaciones que dividen una serie en modos, o Funciones Modales Intrínsecas (FMIs, o IMFs por las siglas en inglés de Intrinsic Mode Functions) sin salir del dominio del tiempo. Se puede comparar con otros métodos de análisis tiempo-espacio como la transformada de Fourier y la descomposición de ondas. La DME ha sido ampliamente aplicada en distintos campos de la ciencia con fines de reconocimiento [11], análisis [36], filtrado [1], predicción [10, etcétera.

\subsection{Redes neuronales artificiales}

Una RNA es una estructura que consiste en un número de nodos conectados a través de conexiones direccionales. Cada nodo representa una unidad de procesamiento, y las conexiones entre los nodos especifican la relación causal entre los nodos conectados. El objetivo principal de una RNA es el de imitar la sinapsis generada en las neuronas, las cuales son la unidad fundamental del sistema nervioso y se encuentran conformadas por un núcleo y un sistema de entradas y salidas denominadas dendritas y axones, respectivamente [5]. Una red neuronal se puede considerar como un procesador distribuido masivamente paralelo que tiene una propensión natural para almacenar conocimiento experimental y ponerlo a disposición para su uso [20]. Su principal similitud con el cerebro humano 
Sistema híbrido basado en redes neuronales artificiales y descomposición modal empírica ...

se debe a que a través de un proceso de aprendizaje, la red es capaz de adquirir conocimiento mismo que almacena en los denominados pesos sinápticos. Algunas de las ventajas de las RNAs que las distinguen de los métodos computacionales convencionales son la manera directa en la que adquieren información acerca del problema mediante la etapa de entrenamiento, su capacidad para trabajar con datos tanto analógicos como discretos, su robustez, su tendencia a describir comportamientos no lineales, etc. 35]

\section{Desarrollo y resultados}

Utilizando los datos de IST mostrados en la Fig. 2, se aplicó DME para encontrar las distintas descomposiciones de los datos correspondientes a distintas aportaciones de frecuencia (Fig. 3). Los datos de IST originales se muestran indicados como puntos en color azul; las descomposiciones modales empíricas se muestran como líneas continuas. La línea negra indica la componente de menor frecuencia, $D_{1}(t)$, mientras que las siguientes dos líneas, roja y azul, indican las siguientes componentes de mayor frecuencia $D_{2}(t)$ y $D_{3}(t)$ respectivamente.

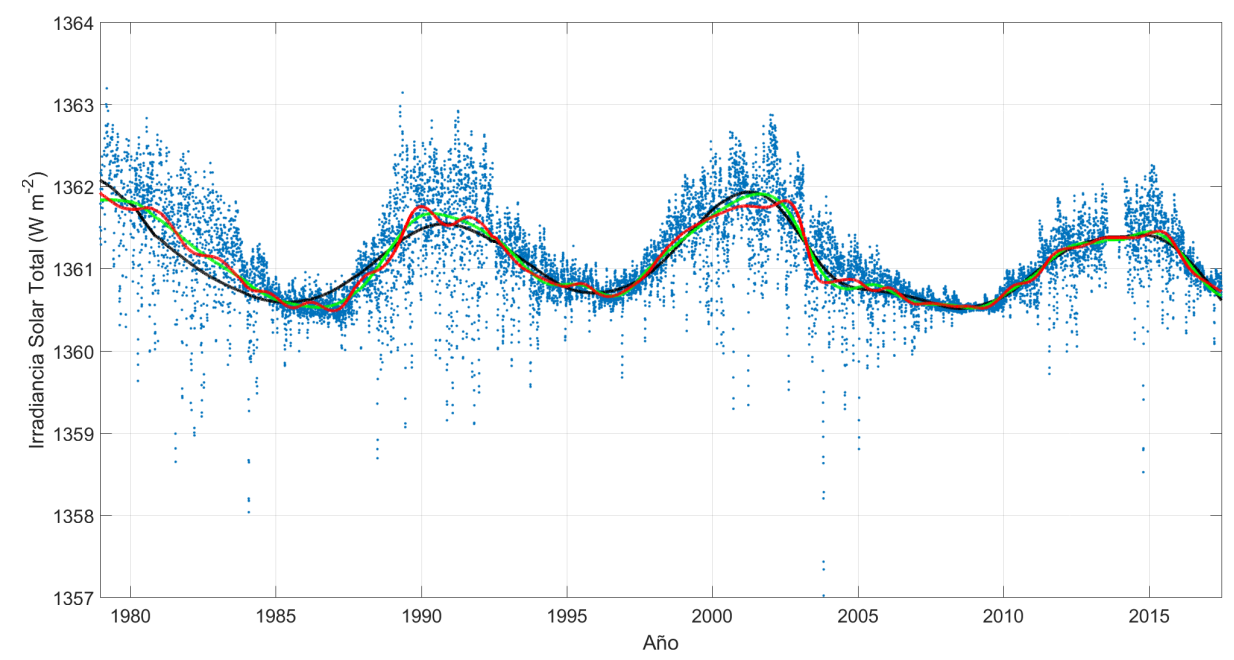

Fig. 3. Descomposición Modal Empírica de los datos de IST (Fig. 2). La línea negra indica la componente de menor frecuencia, $D_{1}(t)$, mientras que las siguientes dos líneas, roja y azul, indican las siguientes componentes de mayor frecuencia, $D_{2}(t)$ y $D_{3}(t)$ respectivamente

Una vez obtenidas las curvas representativas de la DME, partimos de la hipótesis de que el índice de TSM está relacionado con estas decomposiciones, 
pero no de manera suficiente con los datos temporales, es decir, no está únicamente relacionado con su distribución en el tiempo, sino también con su comportamiento creciente/decreciente y su comportamiento cóncavo hacia arriba/abajo. En este sentido es entonces necesario calcular las derivadas y segundas derivadas numéricas de $D_{1}(t), D_{2}(t)$ y $D_{3}(t)$. De esta manera, un vector de entrada para el entrenamiento de la red neuronal se construye al calcular $D_{i}\left(t_{0}\right), D_{i}{ }^{\prime}\left(t_{0}\right)$ y $D_{i}{ }^{\prime \prime}\left(t_{0}\right)$ para cada una de las tres descomposiciones (para $i=1,2,3$ ), es decir, la RNA es entrenada utilizando vectores de entrada de 9 elementos. Cabe destacar que aunque pareciera limitado considerar únicamente tres entradas para determinar el vector de rasgos de cada curva representantiva obtenida mediante la descomposición modal, ésta última evidenció la naturaleza periódica (tipo sinusoidal) de las curvas, lo que garantiza que estos tres elementos contienen una parte muy importante de la información respectiva de cada curva. Adicionalmente, el desempeño de la RNA con vectores de rasgo de esta longitud resulta ser adecuado en cuanto a recursos computacionales bajos para su entrenamiento y ejecución.

Con respecto a las salidas deseadas de la RNA, relacionamos cada una de las entradas (formadas por los 9 elementos descritos anteriormente) para un tiempo $t_{0}$ con el dato correspondiente a la TSM en ese mismo tiempo (1 salida). Para entrenar la RNA utilizamos datos desde 1978 hasta 2005 (70\% de los datos). El resto de los datos, desde 2005 hasta 2016 (30\%), fueron separados del entrenamiento para ser utilizados como datos de validación. Al usar distintos conjuntos de entrenamiento y de validación, verificamos que la RNA está aprendiendo un patrón, y no únicamente memorizando las entradas de entrenamiento con sus respectivas salidas deseadas. La estructura básica de la RNA es una red neuronal prealimentada (feedforward) con tres tipos de capas: entrada, oculta y salida, como se muestra en la Fig. 4. Los parámetros de la RNA se muestran en la Tabla 1. La red entrenada se probó con los conjuntos de entrenamiento y de validación (desde 1978 hasta 2016); los resultados se muestran en la Fig. 4. En esta gráfica se muestran los datos correspondientes a las temperaturas superficiales del mar con la línea azul, y los datos obtenidos de la RNA se muestran con puntos, rojos correspondientes a los datos del conjunto de entrenamiento, y verdes correspondientes a los datos del conjunto de validación. El desempeño de la RNA durante el entrenamiento puede observarse en la Fig. 5.

Tabla 1. Parámetros utilizados en la red neuronal prealimentada.

\begin{tabular}{ll}
\hline Característica & Valor/parámetro \\
\hline Capas de salida & 1 \\
Capas ocultas & 10 \\
Capas de entrada & 1 \\
Tasa de aprendizaje & 0.05 \\
Algoritmo de aprendizaje & Levenberg-Marquardt \\
Error & Error cuadrático medio \\
\hline
\end{tabular}




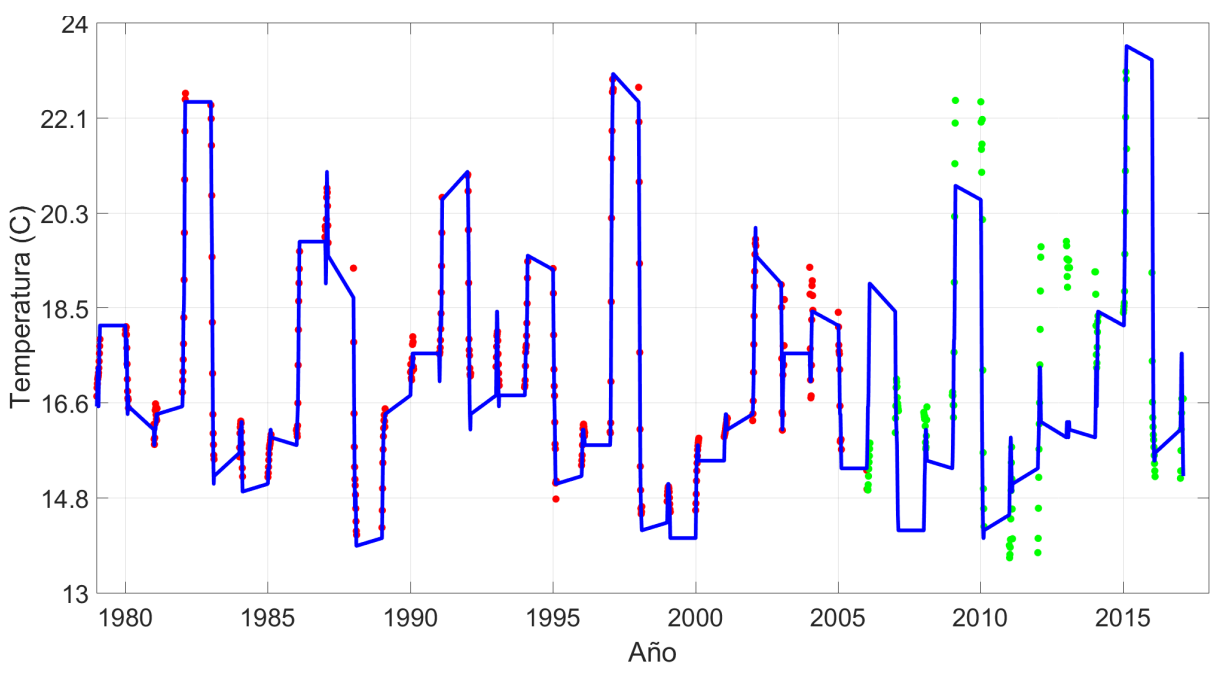

Fig. 4. Los datos de TSM (línea continua en azul) junto con la salida de la RNA para los mismos valores de tiempo. Las salidas correspondientes a datos utilizados para el entrenamiento de la red se muestran como puntos rojos, mientras que aquellos utilizados para la validación se muestran como puntos verdes.

De las Figs. 4 y 5 , es evidente apreciar la efectividad de la red para relacionar los datos de IST a través de sus descomposiciones obtenidas mediante DME y sus respectivas derivadas con los datos de TSM, un indicador directamente relacionado con el calentamiento global. Sin embargo, es evidente también de ambas figuras que los datos de TSM y los datos de salida de validación de la RNA difieren en mayor medida en comparación con aquellos utilizados para su entrenamiento. En este sentido, es importante apreciar que el comportamiento general de ambos conjuntos de datos es muy similar, es decir, los datos de la RNA crecen y decrecen al mismo tiempo que lo hacen los datos de TSM, aunque en distinta magnitud. Como parte del trabajo, se considerarán distintas arquitecturas y configuraciones de la RNA para reducir la discrepancia entre los datos de TSM y los datos de salida de validación.

\section{Conclusiones}

En este trabajo se presenta el diseño y los resultados preliminares de un sistema híbrido basado en DME y en una RNA para relacionar datos de IST con el calentamiento global mediante el indicador de la TSM. Inicialmente, los datos de IST son completados mediante la técnica geoestadística de kriging, para posteriormente ser sujetos a un análisis de DME para obtener sus distintas descomposiciones modales, correspondientes a distintos valores de frecuencia. Estas descomposiciones, junto con sus primeras y segundas derivadas numéricas 


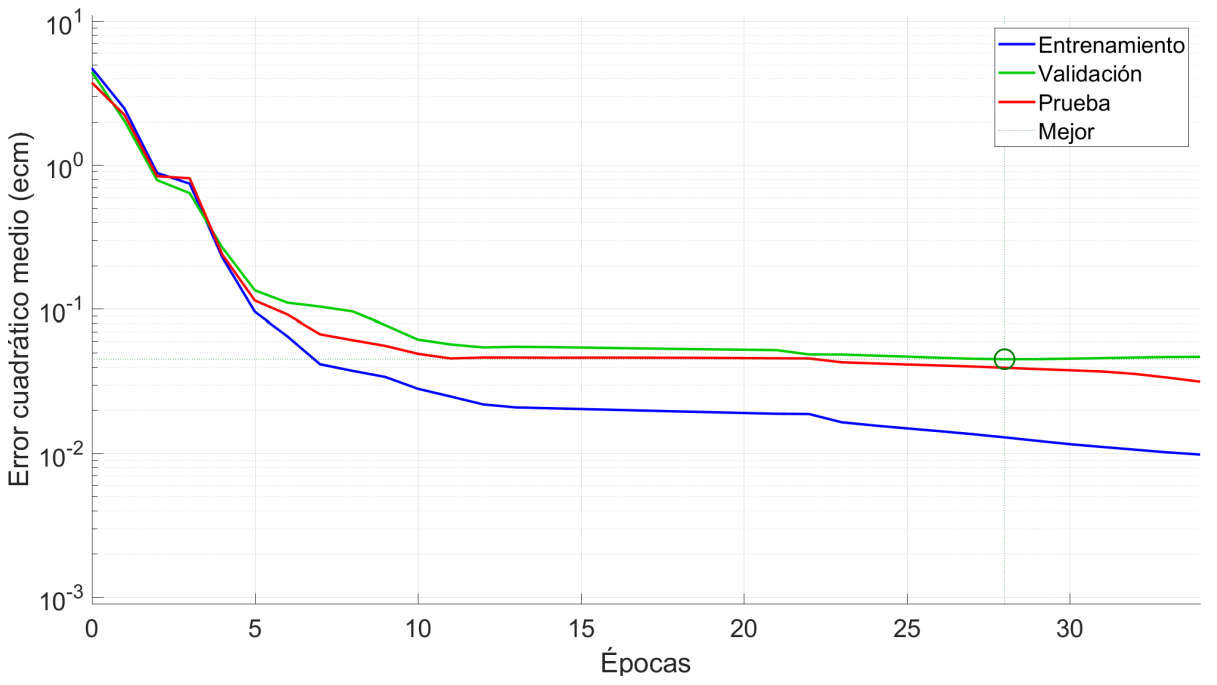

Fig. 5. Desempeño de la RNA durante 34 épocas de entrenamiento. Se muestra el error cuadrático medio para los conjuntos de entrenamiento, validación, prueba. Se indica también el mejor valor alcanzado para el conjunto de validación.

que representan periodos crecientes/decrecientes y con concavidad hacia arriba/abajo, respectivamente, forman al conjunto de entrenamiento de una RNA. Las salidas deseadas de la red fueron asignadas a los distintos valores de TSM. Después de asignar el $70 \%$ del conjunto de datos de entrada al entrenamiento, y el $30 \%$ a la validación, se entrena exitosamente a la RNA de tal manera que nos permite observar una evidente relación entre los datos de IST las variaciones en la TSM.

Con respecto al calentamiento global, los océanos son los mayores responsables de la retención de calor en el sistema Tierra, ya que el calor específico del agua es mucho mayor que el del aire. En este sentido, a pesar de la influencia de materia orgánica y gases de efecto invernadero en las capacidades térmicas del mar 25, este estudio demuestra que las principales variaciones en la TSM se deben a un factor externo como lo es la variabilidad solar.

Mas aún, con los resultados de este estudio, es posible suponer una posible relación entre la IST y el Fenómeno de El Niño. El Fenómeno de El Niño/Oscilación Meridional (ENOM) es uno de los fenómenos climáticos más intrigantes y socioeconómicamente dañinos cuyo origen es aún incierto. Investigación reciente ha apuntado ciertas correlaciones entre el fenómeno ENOM y el cambio climático 3, 15, 49].

Incluso, el IPCC ha sugerido que el cambio climático conllevará a episodios de ENOM cada vez más largos y profundos, los denominados mega-ENOMs 25 27]. Y como uno de los indicadores fundamentales para determinar los periodos de ENOM es la TSM, será interesante explorar, mediante técnicas de inteligencia 
Sistema híbrido basado en redes neuronales artificiales y descomposición modal empírica ...

artificial, la relación entre los indicadores aquí estudiados y otros indicadores fundamentales propios del ENOM.

\section{Trabajo a futuro}

A pesar de ser éste un trabajo aún en proceso, los resultados son claramente alentadores. La relación evidenciada entre los datos de IST y TSM hace de este trabajo el primer paso para la elaboración de un sistema de predicción altamente complejo sobre el calentamiento global con los datos de IST más susceptibles a ajustes y consecuentes predicciones $7,18,42,47$.

El próximo paso para el diseño de este sistema consiste en el ajuste de las descomposiciones modales mediante funciones analíticas, de tal forma que puedan utilizarse para extrapolar las entradas de la RNA, y sea posible obtener predicciones del comportamiento de la TSM en el futuro a corto, mediano y largo plazo, permitiendo evaluaciones más precisas sobre calentamiento global, y potencialmente del fenómeno ENOM.

Agradecimientos. Se agradece al Tecnológico Nacional de México/I.T. Mérida por el apoyo económico mediante los proyectos 6513.18-P y 6511.18-P. Los autores también agradecen el apoyo económico parcial de los proyectos 20181139 , 20180472, 20181441, 20181028 y 20181141, así como al EDI, todos provistos por $\mathrm{SIP} / \mathrm{IPN}$

\section{Referencias}

1. Andrade, A.O., Nasuto, S., Kyberd, P., Sweeney-Reed, C.M., Van Kanijn, F.: EMG signal filtering based on empirical mode decomposition. Biomedical Signal Processing and Control 1(1), 44-55 (2006)

2. Andriopoulou, M., Mavromichalaki, H., Preka-Papadema, P., Plainaki, C., Belov, A., Eroshenko, E.: Solar activity and the associated ground level enhancements of solar cosmic rays during solar cycle 23. Astrophys. Sp. Sci. Trans. 7, 439-443 (2011)

3. Ashok, K., Yamagata, T.: Climate change: The El Niño with a difference. Nature 461(7263), 481 (2009)

4. Attia, A.F., Ismail, H.A., Basurah, H.M.: A Neuro-Fuzzy modeling for prediction of solar cycles 24 and 25. Astrophysics and Space Science 344(1), 5-11 (2013)

5. Basogain Olabe, X.: Redes neuronales artificiales y sus aplicaciones. Med. Intensiva 29, 13-20 (2005)

6. Belov, A., Eroshenko, E., Kryakunova, O., Kurt, V., Yanke, V.: Ground level enhancements of solar cosmic rays during the last three solar cycles. Geomagnetism and Aeronomy 50(1), 21-33 (2010)

7. Calvo, R., Ceccato, H., Piacentini, R.: Neural network prediction of solar activity. The Astrophysical Journal 444, 916-921 (1995)

8. Claussen, M., Mysak, L., Weaver, A., Crucifix, M., Fichefet, T., Loutre, M.F., Weber, S., Alcamo, J., Alexeev, V., Berger, A., et al.: Earth system models of intermediate complexity: closing the gap in the spectrum of climate system models. Climate dynamics 18(7), 579-586 (2002) 
9. Coddington, O., Lean, J., Lindholm, D., Pilewskie, P., Snow, M., NOAA CDR Program: NOAA Climate Data Record (CDR) of Total Solar Irradiance (TSI), NRLTSI Version 2. Daily record. https://www.ngdc.noaa.gov/docucomp/ page? $\mathrm{xml}=\mathrm{NOAA} / \mathrm{NESDIS} / \mathrm{NCDC} / \mathrm{Geoportal} / \mathrm{iso} / \mathrm{xml} / \mathrm{C} 00828 . \mathrm{xml} \& \mathrm{view}=$ getDataView\&header $=$ none $(2015)$, last accessed 2018/01/06

10. Drakakis, K.: Empirical mode decomposition of financial data. Int. Math. Forum 4, 1191-1202 (2008)

11. Du, H.k., Cao, J.x., Xue, Y.j., Wang, X.j.: Seismic facies analysis based on selforganizing map and empirical mode decomposition. Journal of Applied Geophysics $112,52-61(2015)$

12. Flato, G.M.: Earth system models: an overview. Wiley Interdisciplinary Reviews: Climate Change 2(6), 783-800 (2011)

13. Ganopolski, A., Petoukhov, V., Rahmstorf, S., Brovkin, V., Claussen, M., Eliseev, A., Kubatzki, C.: CLIMBER-2: a climate system model of intermediate complexity. Part II: model sensitivity. Climate Dynamics 17(10), 735-751 (2001)

14. Gazis, P., Richardson, J., Paularena, K.: Long term periodicity in solar wind velocity during the last three solar cycles. Geophysical research letters 22(10), 1165-1168 (1995)

15. Gergis, J.L., Fowler, A.M.: A history of ENSO events since AD 1525: implications for future climate change. Climatic Change 92(3-4), 343-387 (2009)

16. Goosse, H., Brovkin, V., Fichefet, T., Haarsma, R., Huybrechts, P., Jongma, J., Mouchet, A., Selten, F., Barriat, P.Y., Campin, J.M., et al.: Description of the Earth system model of intermediate complexity LOVECLIM version 1.2. Geoscientific Model Development 3, 603-633 (2010)

17. Hale, G.E.: Sun-spots as magnets and the periodic reversal of their polarity. Nature 113(2829), 105 (1924)

18. Hathaway, D.H., Wilson, R.M., Reichmann, E.J.: A synthesis of solar cycle prediction techniques. Journal of Geophysical Research: Space Physics 104(A10), 22375-22388 (1999)

19. Hawkins, E., Sutton, R.: The potential to narrow uncertainty in regional climate predictions. Bulletin of the American Meteorological Society 90(8), 1095-1107 (2009)

20. Hayati, M., Mohebi, Z.: Application of artificial neural networks for temperature forecasting. World Academy of Science, Engineering and Technology 28(2), 275-279 (2007)

21. Helama, S., Fauria, M.M., Mielikäinen, K., Timonen, M., Eronen, M.: SubMilankovitch solar forcing of past climates: mid and late Holocene perspectives. Bulletin 122(11-12), 1981-1988 (2010)

22. Huang, N.E., Shen, Z., Long, S.R., Wu, M.C., Shih, H.H., Zheng, Q., Yen, N.C., Tung, C.C., Liu, H.H.: The empirical mode decomposition and the Hilbert spectrum for nonlinear and non-stationary time series analysis. In: Proceedings of the Royal Society of London A: mathematical, physical and engineering sciences. vol. 454, pp. 903-995. The Royal Society (1998)

23. Huntingford, C., Lowe, J., Booth, B.B., Jones, C.D., Harris, G., Gohar, L., Meir, P.: Contributions of carbon cycle uncertainty to future climate projection spread. Tellus B 61(2), 355-360 (2009)

24. Intergubernamental Panel on Climate Change: Homepage. http://www.ipcc.ch/ home_languages_main_spanish.shtml (2014), last accessed 2018/04/01

25. Interngubernamental Panel on Climate Change: IPCC: Working Group I Contribution to the IPCC Fifth Assessment Report, Climate Change 2013: The Physical Science Basis. IPCC. AR5. Interngubernamental Panel on Climate Change (2014) 
Sistema híbrido basado en redes neuronales artificiales y descomposición modal empírica ...

26. Kane, R.: Short-term periodicities in solar indices. Solar Physics 227(1), 155-175 (2005)

27. Kim, B.H., Ha, K.J.: Observed changes of global and western Pacific precipitation associated with global warming SST mode and mega-ENSO SST mode. Climate dynamics 45(11-12), 3067-3075 (2015)

28. Kim, D., Oh, H.S.: EMD: a package for empirical mode decomposition and Hilbert spectrum. The R Journal 1(1), 40-46 (2009)

29. Kopp, G., Lean, J.L.: A new, lower value of total solar irradiance: Evidence and climate significance. Geophysical Research Letters 38(1) (2011)

30. Krige, D.: A statistical approach to some basic mine valuation problems on the witwatersrand. J. Chem. Metall. Min. Soc. S. Afr. p. 201-215 (1952)

31. Laprise, R.: Regional climate modelling. Journal of Computational Physics 227(7), 3641-3666 (2008)

32. Liu, L., Cheng, Y., Wang, X.: Genetic algorithm optimized Taylor Kriging surrogate model for system reliability analysis of soil slopes. Landslides $14(2), 535-546$ (2017)

33. Matheron, G.: Principles of geostatistics. Economic geology 58(8), 1246-1266 (1963)

34. Mursula, K., Zieger, B.: The 1.3-year variation in solar wind speed and geomagnetic activity. Advances in Space Research 25(9), 1939-1942 (2000)

35. Nannariello, J., Fricke, F.: Introduction to neural network analysis and its application to building services engineering. Building Services Engineering Research and Technology 22(1), 58-68 (2001)

36. Nunes, J.C., Bouaoune, Y., Delechelle, E., Niang, O., Bunel, P.: Image analysis by bidimensional empirical mode decomposition. Image and vision computing 21(12), 1019-1026 (2003)

37. Obridko, V., Shelting, B.: Occurrence of the 1.3-year periodicity in the large-scale solar magnetic field for 8 solar cycles. Advances in Space Research 40(7), 1006-1014 (2007)

38. Orozco-Del-Castillo, M., Ortiz-Alemán, J., Couder-Castañeda, C., HernándezGómez, J., Solís-Santomé, A.: High solar activity predictions through an artificial neural network. International Journal of Modern Physics C 28(06), 1750075 (2017)

39. Petoukhov, V., Claussen, M., Berger, A., Crucifix, M., Eby, M., Eliseev, A., Fichefet, T., Ganopolski, A., Goosse, H., Kamenkovich, I., et al.: EMIC Intercomparison Project (EMIP-CO 2): comparative analysis of EMIC simulations of climate, and of equilibrium and transient responses to atmospheric CO 2 doubling. Climate Dynamics 25(4), 363-385 (2005)

40. Petrovay, K.: Solar cycle prediction. Living reviews in solar physics 7(1), 6 (2010)

41. Prince, A.M., Thomas, S., Jon, R., Jayapandian, D.: A study on midrange periodicity of sunspot number during solar cycles 21, 22, 23 \& 24. Int. J. Sci. Res. Publ. 3, 1-5 (2013)

42. Rigozo, N., Echer, M.S., Evangelista, H., Nordemann, D., Echer, E.: Prediction of sunspot number amplitude and solar cycle length for cycles 24 and 25. Journal of Atmospheric and Solar-Terrestrial Physics 73(11-12), 1294-1299 (2011)

43. Ritz, S.P., Stocker, T.F., Joos, F.: A coupled dynamical ocean-energy balance atmosphere model for paleoclimate studies. Journal of Climate 24(2), 349-375 (2011)

44. Rummukainen, M.: State-of-the-art with Regional Climate Models. Wiley Interdisciplinary Reviews: Climate Change 1(1), 82-96 (2010) 
45. Shaffer, G., Malskær Olsen, S., Pepke Pedersen, J.: Presentation, calibration and validation of the low-order, DCESS Earth System Model (Version 1). Geoscientific Model Development 1(1), 17-51 (2008)

46. Shea, M., Smart, D.: A summary of major solar proton events. Solar Physics 127(2), 297-320 (1990)

47. Uwamahoro, J., McKinnell, L.A., Cilliers, P.J.: Forecasting solar cycle 24 using neural networks. Journal of Atmospheric and Solar-Terrestrial Physics 71(5), 569$574(2009)$

48. Weaver, A.J., Eby, M., Wiebe, E.C., Bitz, C.M., Duffy, P.B., Ewen, T.L., Fanning, A.F., Holland, M.M., MacFadyen, A., Matthews, H.D., et al.: The UVic Earth System Climate Model: Model description, climatology, and applications to past, present and future climates. Atmosphere-Ocean 39(4), 361-428 (2001)

49. Xie, S.P., Deser, C., Vecchi, G.A., Collins, M., Delworth, T.L., Hall, A., Hawkins, E., Johnson, N.C., Cassou, C., Giannini, A., et al.: Towards predictive understanding of regional climate change. Nature Climate Change 5(10), 921 (2015)

50. Zhang, J., Li, X., Yang, R., Liu, Q., Zhao, L., Dou, B.: An extended Kriging method to interpolate near-surface soil moisture data measured by wireless sensor networks. Sensors 17(6), 1390 (2017)

51. Zickfeld, K., Eby, M., Weaver, A.J., Alexander, K., Crespin, E., Edwards, N.R., Eliseev, A.V., Feulner, G., Fichefet, T., Forest, C.E., et al.: Long-term climate change commitment and reversibility: an EMIC intercomparison. Journal of Climate 26(16), 5782-5809 (2013) 